\title{
Ultrafast Stretched-Pulse Fiber Laser Mode-Locked by Carbon Nanotubes
}

\author{
Zhipei Sun, Tawfique Hasan, Fengqiu Wang, Aleksey G. Rozhin, Ian H. White, and Andrea C. Ferrari ( $\square)$ \\ Department of Engineering, University of Cambridge, Cambridge CB3 OFA, UK \\ Received: 21 February 2010 / Revised: 3 April 2010 / Accepted: 4 April 2010 \\ (C) The Author(s) 2010. This article is published with open access at Springerlink.com
}

\begin{abstract}
Ultrafast fiber sources having short pulses, broad bandwidth, high energy, and low amplitude fluctuations have widespread applications. Stretched-pulse fiber lasers, incorporating segments of normal and anomalous dispersion fibers, are a preferred means to generate such pulses. We realize a stretched-pulse fiber laser based on a nanotube saturable absorber, with $113 \mathrm{fs}$ pulses, $33.5 \mathrm{~nm}$ spectral width and $\sim 0.07 \%$ amplitude fluctuation, outperforming current nanotube-based designs.
\end{abstract}

\section{KEYWORDS}

Nanotubes, fiber laser, saturable absorber, ultrafast pulse generation

\section{Introduction}

Passively mode-locked fiber lasers are attractive for many applications, such as optical communications [1], spectroscopy [2], and biomedical diagnostics [3], because of their simplicity, compactness, efficient heat dissipation, and ability to generate high-quality pulses [3-5]. The dominant mode-locking technology is based on semiconductor saturable mirrors (SESAMs) [6]. However, SESAMs are typically complex quantum wells, fabricated by molecular beam epitaxy on distributed Bragg reflectors $[1,3,6]$, and ion implantation is normally required to reduce the recovery time [6]. Therefore, novel saturable absorbers with better performance, cheaper fabrication and easier integration are of great interest. Alternative saturable absorbers based on single wall carbon nanotubes (SWNTs) and graphene are at the centre of an intense research effort $[7,8]$, due to their broad operation range, low saturation power, easy fabrication, mechanical and environmental robustness, and quick recovery times [7-28]. These have been used to mode-lock fiber [8-23], waveguide [24], solid-state [25-27], and semiconductor lasers [28]. Present mode-locking technology typically relies on soliton-like operation. In this regime, nonlinear effects due to strong mode confinement $[5,29,30]$, and the long fibre required (typically $\sim 10 \mathrm{~m}[5,30]$ ), distort the transform-limited soliton pulses, and limit the generation of high-energy pulses $[5,29,30]$. The resulting pulses normally have strong sidebands, in both frequency and time domains $[5,29,30]$. Indeed, the shortest pulse to date using a SWNT-based modelocker at $1550 \mathrm{~nm}$ is $\sim 115 \mathrm{fs}$, with traditional soliton-like operation [22], but with strong sidebands in the spectral and time domains [22] and random multiple pulses $[5,12,22,29,30]$, limiting the achievement of ever shorter pulses, clean spectra, low amplitude fluctuations and higher energy.

$\overline{\text { Address correspondence to acf26@ }}$ cam.ac.uk 
To overcome these restrictions, a dispersion management design utilizing different types of fibers with alternating anomalous and normal group velocity dispersion (GVD) (i.e., group velocity decreasing and increasing, respectively, with decreasing optical frequency) has been proposed [5, 29, 30]. Dispersion plays a critical role in the propagation of ultrafast pulses within optical fibers, as different spectral components move at different speed. In principle, dispersion-induced pulse broadening could be compensated by an equal amount of dispersion of opposite sign. As indicated in Fig. 1, alternating normal and anomalous GVD allows periodic stretching and compression of the intracavity pulses in the resonator $[5,29,30]$. The average pulse width in one cavity round trip can increase by an order of magnitude or more $[5,29,30]$. This significantly decreases the intracavity average peak power, compared to soliton-like operation. Thus, nonlinear optical effects triggered by high intensity are mitigated. This allows high quality pulses with larger energy (up to nanojoules) to be achieved for a specific system-bearable nonlinearity [5, 29-31]. This configuration is known as the stretched-pulse design. Ultrafast high-energy fibre lasers enabled by the stretched-pulse design have a variety of potential applications, such as amplification, frequency doubling, and spectroscopy $[4,5,32,33]$.

Here, we demonstrate the stretched-pulse fiber design for a laser using a SWNT saturable absorber. We achieve $113 \mathrm{fs}$ pulses at $1.56 \mu \mathrm{m}$, with $18.76 \mathrm{MHz}$
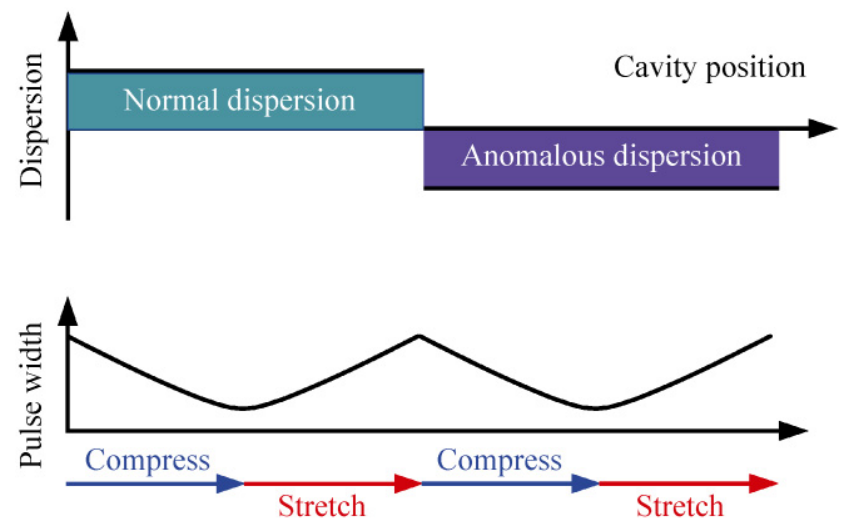

Figure 1 Simplified scheme of a stretched-pulse laser, with the dispersion map (top) and pulse width distribution (bottom). Normal (positive) and anomalous (negative) dispersion are marked with different colours repetition rate, $\sim 33.5 \mathrm{~nm}$ output spectral width, and $\sim 0.07 \%$ fluctuation.

\section{Experimental}

\subsection{Device fabrication and characterization}

Pristine SWNTs cannot be stably dispersed in a highly polar solvent like water without functionalization [34]. Usually, surfactants [34-37] or water soluble polymers [37-39] are used to exfoliate SWNTs from their naturally occurring bundles. The most commonly used surfactants are sodium dodecyl sulfate (SDS) and sodium dodecyl benzenesulfonate (SDBS) [34-37]. Both are linear chain anionic surfactants with a hydrophobic tail and a hydrophilic head [35]. When SWNTs are isolated by the shear forces from the formation and collapse of cavities generated by ultrasounds [40], surfactants-at concentrations above the critical micelle concentration-form micelles around individual SWNTs and small SWNT bundles, preventing their re-aggregation [34].

To make our polyvinyl alcohol (PVA)-SWNT saturable absorber composite, we use SDBS due to its efficiency in dispersing large amounts of SWNTs [35-37]. Importantly, it disperses SWNTs with a larger diameter range compared to bile salts, the latter being more effective with small diameter tubes [7]. The amounts of SWNTs and dispersant used to prepare solutions vary according to the SWNT purity. For the PVA-SWNT composites, we use SWNTs grown by laser ablation, as described in Ref. [41], and purified as reported in Ref. [42]. The mean diameter $(1.3 \mathrm{~nm})$ of the SWNTs used here ensures that the semiconducting SWNTs in the sample have an absorption band at around $1500 \mathrm{~nm}$. We dispersed $2 \mathrm{mg}$ of SWNTs in $14 \mathrm{~mL}$ of deionized water with $40 \mathrm{mg}$ of SDBS for $2-3 \mathrm{~h}$ at $10-12{ }^{\circ} \mathrm{C}$ using a Bioruptor (Diagenode) sonicator system operating at $20 \mathrm{kHz}$ with a power of $270 \mathrm{~W}$. This resulted in a dark dispersion with no visible SWNT aggregates, Fig. 2(a). Note that the actual power delivered to the sample is substantially lower than $270 \mathrm{~W}$ due to the non-contact operation of this ultrasonicator. The residual bundles and impurities were removed by ultracentrifugation at $\sim 80000 \mathrm{~g}$ for $1 \mathrm{~h}$ by using a fixed angle MLA- 80 rotor in an 
Optima-Max-E ultracentrifuge (Beckman Coulter). The supernatant was then decanted. The absorption of the SWNT dispersion at $1550 \mathrm{~nm}$ was 0.15 for a $10 \mathrm{~mm}$ absorption path length. The dispersion was then mixed with a $20 \mathrm{wt} \%$ aqueous PVA solution. Ultrasonicating the mixture resulted in a uniform solution which was then drop-cast on a Petri dish. Slow evaporation (1-2 weeks) under ambient temperature and pressure in a desiccator gave a free standing SWNT-PVA composite, $50 \mu \mathrm{m}$ thick with homogeneously dispersed tubes $[7,11,13]$. The film was then dried at $45^{\circ} \mathrm{C}$. Figure 2(b) shows a micrograph of the composite, confirming the homogeneous nanotube dispersion. Approximately $2 \mathrm{~mm}^{2}$ of the composite were used between fiber connectors for our transmissive-type device configuration, Fig. 2(c).

The SWNT films were characterized by Raman spectroscopy (Fig. 3) and absorption spectrophotometry (Fig. 4). In the $1550-1590 \mathrm{~cm}^{-1}$ region, the Raman spectra of SWNTs have two distinct features, the so-called $\mathrm{G}^{+}$and $\mathrm{G}^{-}$peaks. These originate from the tangential (TO) and the longitudinal (LO) modes resulting from the splitting of the doubly degenerate $E_{2 g}$ phonon of graphene [43]. In metallic tubes, the LO mode is affected by a Kohn anomaly (KA) [43, 44], which causes the softening of this phonon $[43,44]$. Since KA are not present in semiconducting SWNTs, the $\mathrm{G}^{+}, \mathrm{G}^{-}$assignment in metallic SWNTs is the opposite of semiconducting tubes [43]. In semiconducting tubes, both the $\mathrm{G}^{+}$and $\mathrm{G}^{-}$bands appear as sharp Lorentzian peaks. The $\mathrm{G}^{+}$is usually more intense than the $G^{-}$, and its position is nearly independent of tube diameter, whereas the $\mathrm{G}^{-}$position decreases with decreasing tube diameter. On the other hand, in

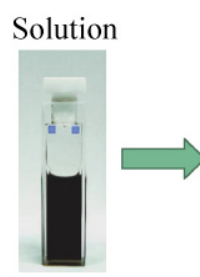

(a)
Composite

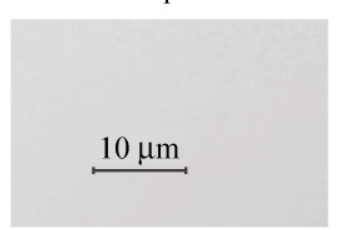

(b)

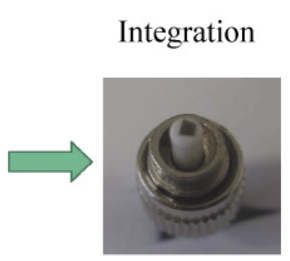

(c)
Figure 2 Preparation and integration of the SWNT-PVA composite: (a) photograph of the SWNT dispersion after ultrasonication; (b) optical micrograph of the SWNT-PVA composite showing no aggregation; (c) integration of $\sim 2 \mathrm{~mm}^{2}$ of the composite to a fiber connector metallic tubes the $\mathrm{G}^{-}$peak is usually rather intense, very broad, and downshifted with respect to its counterpart in semiconducting tubes [43]. The other prominent features in the Raman spectrum of SWNTs are the radial breathing modes (RBM). These are associated with a symmetric movement of carbon atoms in the radial direction, whose energy is inversely related to the tube diameter. The SWNT diameter, $d$, can be derived from the RBM frequency, $\omega_{\mathrm{RBM}}$, by means of the expression $d=C_{1} /\left(\omega_{\mathrm{RBM}}-C_{2}\right)$, combined with the Kataura plot and the known excitation energy [45-47]. A variety of different values of $C_{1}$ and $C_{2}$ have been proposed [45-47] but their precise value is only critical for chirality assignment [45-47], which is not an issue here, since we are interested only in the diameter. The shapes of the $G$ peaks at the two excitation energies, shown in Fig. 3, imply the presence of both metallic and semiconducting tubes. The RBMs indicate a diameter distribution of 1-1.4 nm, implying a band gap of $0.7-1.1 \mathrm{eV}$ [48]. We target this range to cover the gain bandwidth of our erbium fiber at $1550 \mathrm{~nm}$. This is verified by the absorption spectrum, Fig. 4 , which has a maximum at $\sim 1600 \mathrm{~nm}$ with a bandwidth of $\sim 340 \mathrm{~nm}$.

After packaging (Fig. 2(c)), the nonlinear absorption of the film is characterized by power-dependent absorbance performed with a pulsed erbium-doped fiber (EDF) laser at $1550 \mathrm{~nm}$. This gives a saturation intensity of $\sim 18.9 \mathrm{MW} / \mathrm{cm}^{2}$ and modulation depth

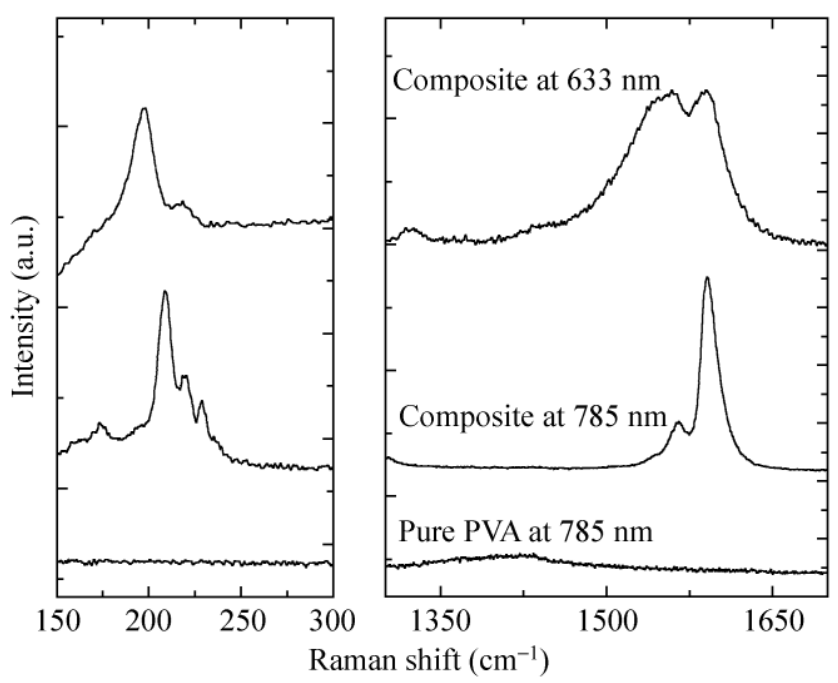

Figure 3 Raman spectra of pure PVA and SWNT-PVA composite measured with excitation wavelengths of 633 and $785 \mathrm{~nm}$ 


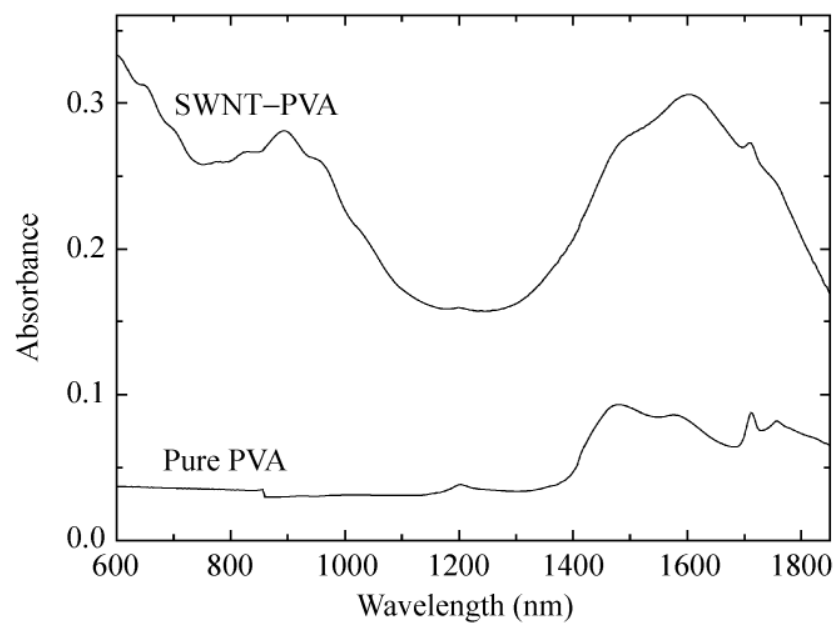

Figure 4 Absorption spectra of the SWNT-PVA composite and pure PVA film

of $\sim 16.9 \%$ (Fig. 5), sufficient to provide strong pulse shaping, reliable self-starting and stabilized pulse generation [6].

\subsection{Laser setup}

The packaged SWNT-PVA composite was inserted in a fiber laser cavity. The laser was in a ring configuration. A $3.6 \mathrm{~m}$ EDF (Fibercore Ltd.) was forward pumped by a $980 \mathrm{~nm}$ diode laser through a fused wavelength division multiplexer to provide gain. To realize the stretched-pulse design at $\sim 1.5 \mu \mathrm{m}$, the EDF was selected with normal dispersion at our operating wavelength, while the other fibers were chosen with anomalous dispersion to set the total intracavity GVD close to zero

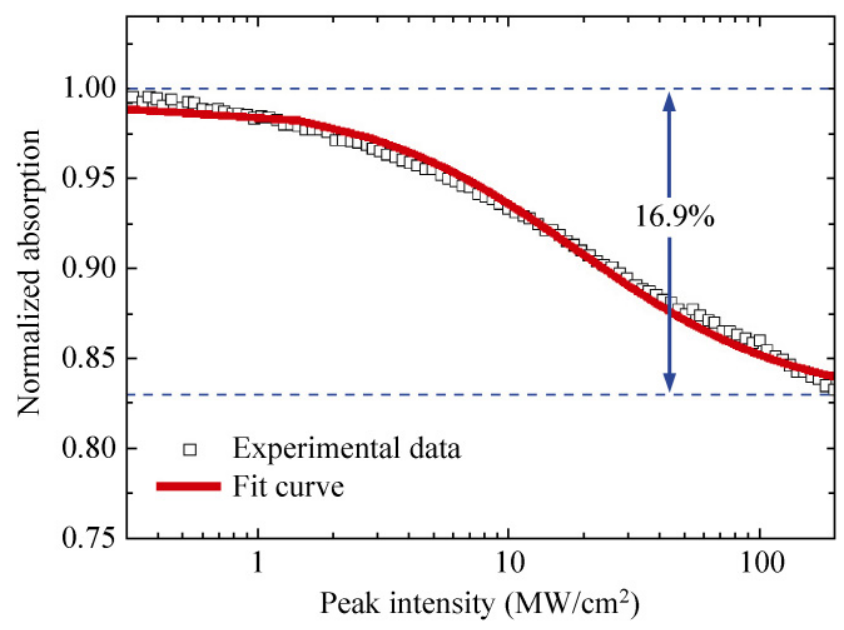

Figure 5 Nonlinear power-dependent absorbance of SWNT-PVA
$[4,5,29-33]$. The GVD was measured by inserting the same EDF into a home-made soliton-like fiber laser. We evaluated the EDF dispersion by measuring the wavelength shift $(\Delta \lambda)$ between sidebands and central wavelength of the soliton pulse spectrum [49]. We obtained a GVD coefficient $\beta_{2} \sim 36 \mathrm{ps}^{2} / \mathrm{km}$. The rest of the laser was constructed from Flex 1060 and Corning SMF-28 fibers, with anomalous dispersion. The total length of Flex $1060\left(\beta_{2}=-7 \mathrm{ps}^{2} / \mathrm{km}\right)$ was $\sim 1.83 \mathrm{~m}$, while for SMF-28 $\left(\beta_{2}=-22 \mathrm{ps}^{2} / \mathrm{km}\right)$ we used $\sim 5.74 \mathrm{~m}$, providing an intracavity GVD $\sim-0.008 \mathrm{ps}^{2} \pm 0.005 \mathrm{ps}^{2}$. The total cavity length was $\sim 11.17 \mathrm{~m}$. A polarizationindependent optical isolator ensured unidirectional operation. For mode-locking optimization, we adjusted the polarization using two intracavity polarization controllers. A fused 20/80 output coupler was utilized. The $20 \%$ port extracted the pulses from the cavity. The pump and average output power were monitored by a photodiode power meter (Ophir PD300-IR). A spectrum analyzer (HP 86140A) with $0.1 \mathrm{~nm}$ resolution and a second harmonic generation (SHG) autocorrelator (Inrad 5-14-LDA) with $\sim 5$ fs resolution were used to measure the output spectrum and pulse duration.

\section{Results and discussion}

Continuous wave operation starts at $\sim 7.5 \mathrm{~mW}$ pump power. Self-starting single-pulse mode-locking is observed at $\sim 12.8 \mathrm{~mW}$. Figure 6 shows a typical output pulse spectrum. The full width at half maximum (FWHM) bandwidth is $\sim 33.5 \mathrm{~nm}$. To the best of our knowledge, this is the widest spectrum achieved thus far from an EDF laser mode-locked by SWNTs. No sidebands are observed. Figure 7 shows a plot of a typical output SHG autocorrelation trace, including Gaussian and sech ${ }^{2}$ fits. A sech ${ }^{2}$ pulse profile is typically expected for soliton fiber lasers. However, a Gaussian also fits well our experimental data, as expected for stretched-pulse lasers [30]. The autocorrelation FWHM is $~ 160 \mathrm{fs}$. Assuming a Gaussian profile, the data deconvolution gives a pulse duration of $\sim 113 \mathrm{fs,}$ the shortest reported to date from an EDF laser mode-locked by SWNTs. The time-bandwidth product (TBP) of the output pulses is $\sim 0.467$, with a minor deviation from the value of 0.44 expected for 


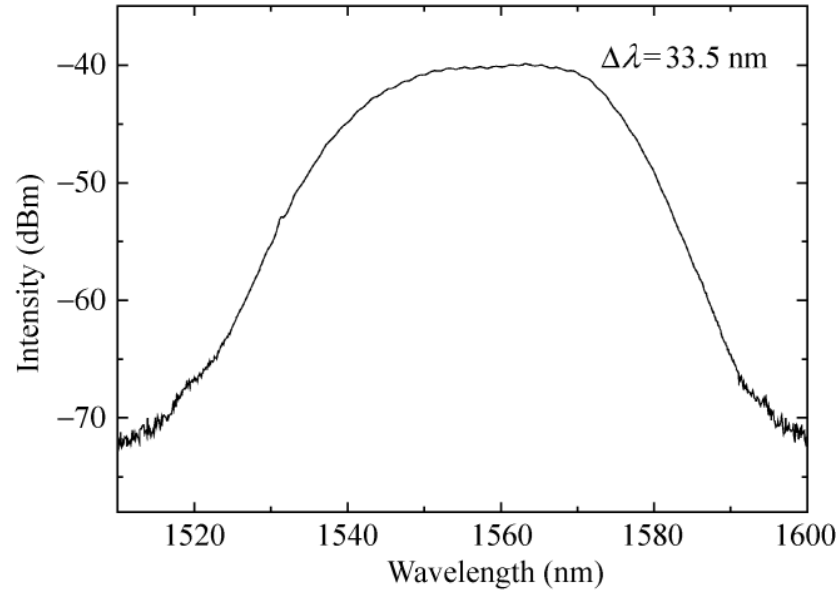

Figure 6 Typical output spectrum, with bandwidth $\sim 33.5 \mathrm{~nm}$

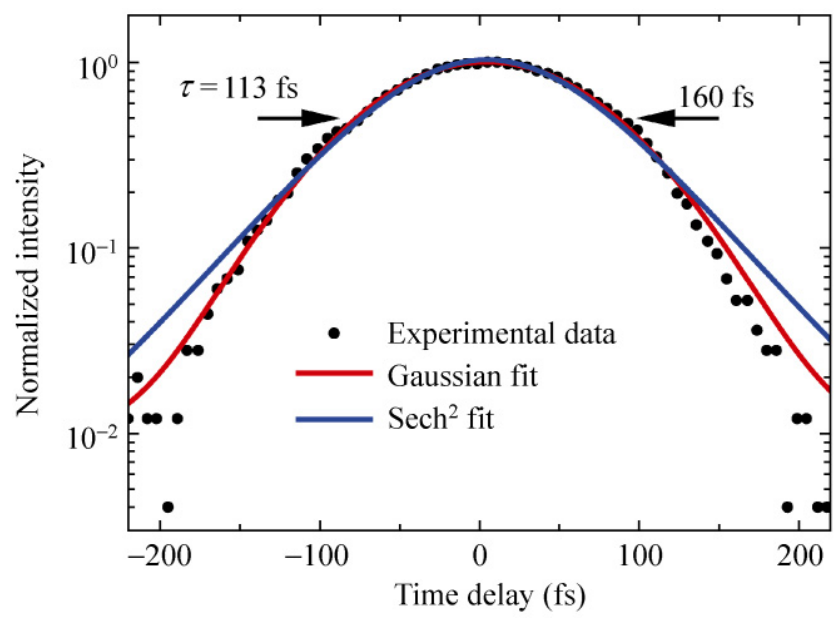

Figure 7 Autocorrelation trace, with Gaussian and $\operatorname{sech}^{2}$ fits

transform-limited Gaussian pulses [50]. This could be caused by uncompensated third-order dispersion, distorting the intracavity pulse [31] and also limiting the minimum achievable pulse width $[51,52]$. Another limitation to shorter pulses could be spectral filtering effects introduced by the erbium gain medium $[5,29]$. Using a $\sim 0.03 \mathrm{ps}^{2}$ fiber patchcord (1.37 m SMF-28), the FWHM of the fitted autocorrelation trace is $\sim 1.358$ ps, corresponding to $\sim 961$ fs for a Gaussian temporal profile. This shows that the output pulse stretches $\sim 8$ times after passing a fiber with $\sim 0.033 \mathrm{ps}^{2}$ GVD. The pulse evolution and the $\sim-0.096 \mathrm{ps}^{2} \mathrm{GVD}$ of the intracavity fibers (3.78 m SMF-28 and $1.83 \mathrm{~m}$ Flexcor 1060) confirm the stretched-pulse design of our laser.

The operating stability is characterized from radiofrequency (RF) spectrum measurements of the output intensity [53]. The repetition rate is $\sim 18.76 \mathrm{MHz}$, corresponding to a $\sim 53.3$ ns round-trip time, Fig. 8 (a). No spectrum modulation is observed over $1.5 \mathrm{GHz}$ (Fig. 8(b)), with no Q-switching instabilities. Figure 9(a) shows a plot of the RF spectrum around the fundamental cavity round-trip frequency. A signalto-noise ratio $\Delta P=\sim 77 \mathrm{~dB}$ (10.7 contrast) is observed, highlighting the low-amplitude fluctuation $\Delta E / E$ of the laser. From $\frac{\Delta E}{E}=\sqrt{\Delta P \times \frac{\Delta f}{\Delta f_{\text {res }}}}$, where $\Delta f$ and $\Delta f_{\text {res }}$ are frequency jitter bandwidth and resolution respectively [53], we estimate an amplitude fluctuation $\Delta E / E \approx 7 \times 10^{-4}$. Figure $9(\mathrm{~b})$ shows a plot of the $R F$ spectrum around the tenth harmonic. $A \sim 60 \mathrm{~dB}$ peakto-background ratio is measured, indicating low timing jitter and good mode-locking stability [53].

Compared with previous SWNT mode-locked EDF lasers [9, 20, 22], our system provides shorter durations, wider and cleaner spectra, and lower amplitude noise fluctuations, thanks to our dispersion-managed stretched-pulse configuration realized by using fibers

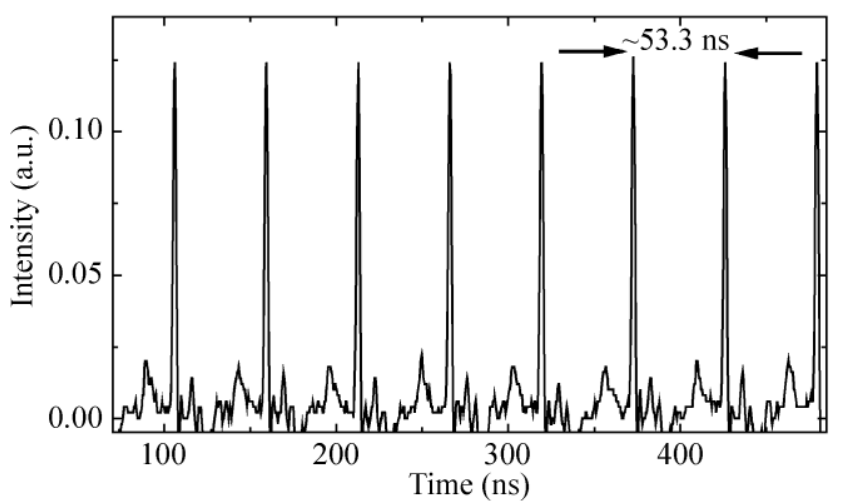

(a)

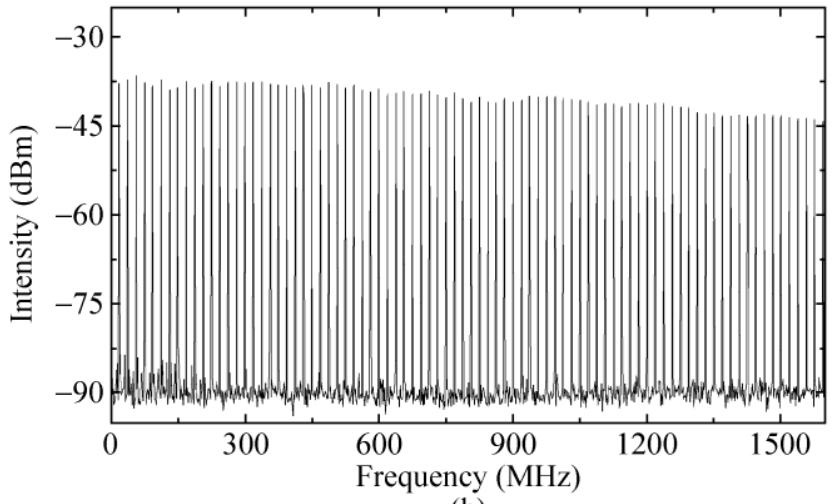

(b)

Figure 8 (a) Oscilloscope trace. (b) RF spectrum up to $1.5 \mathrm{GHz}$ 


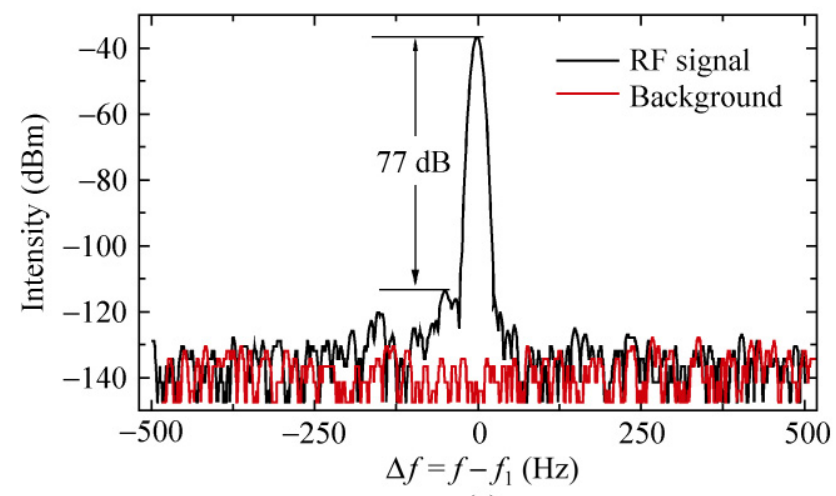

(a)

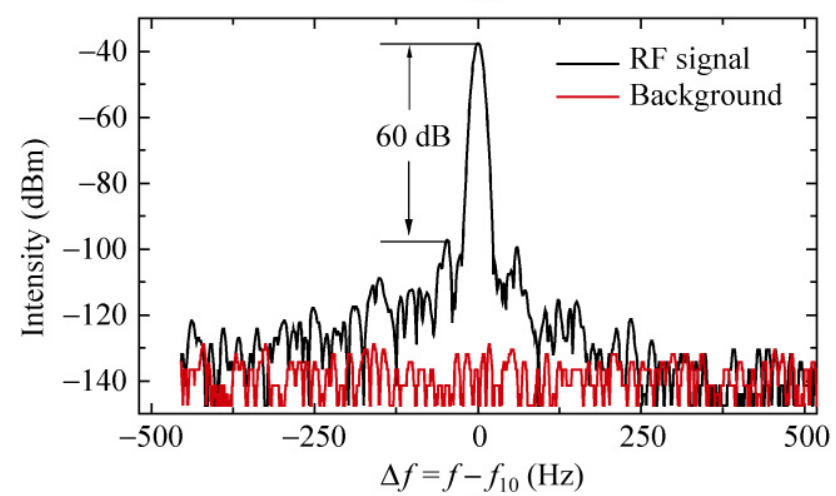

(b)

Figure 9 RF spectra around (a) the fundamental repetition rate $\left(f_{1}=18.76 \mathrm{MHz}\right)$, and (b) the 10 th harmonic repetition rate $\left(f_{10}=\right.$ $187.6 \mathrm{MHz})$

with relatively large normal and anomalous dispersions. In previous pulsed laser designs, the intracavity GVD was typically set as negative with a large absolute value to favour soliton-like pulses [5, 10, 20, 22]. With this design, the width and quality of the output pulses are limited by overdriven nonlinearities and instabilities $[5,29,49]$. In contrast, in our case, nonlinearities significantly decrease, as a result of stretching and compressing the intracavity pulse. Even shorter pulse duration, greater spectral width and higher output power could be achieved by further improvement of the cavity design, employing evanescent field interaction with SWNT saturable absorbers $[12,21]$.

\section{Conclusions}

We demonstrated a stretched-pulse erbium-doped fiber laser using a SWNT-based saturable absorber, generating 113 -fs pulses at $1.56 \mu \mathrm{m}$. The output spectral width is $33.5 \mathrm{~nm}$. This can lead to novel light sources meeting the pulse length, quality and energy requirements for many applications, such as micro-machining and optical tomography, opening new opportunities in metrology, spectroscopy and biomedical diagnostics.

\section{Acknowledgements}

We acknowledge F. Hennrich for providing SWNTs and funding from a Royal Society Brian Mercer Award for Innovation, King's College, Cambridge, ERC grant NANOPOTS, and EPSRC grant EP/G030480/1.

Open Access: This article is distributed under the terms of the Creative Commons Attribution Noncommercial License which permits any noncommercial use, distribution, and reproduction in any medium, provided the original author(s) and source are credited.

\section{References}

[1] Agrawal, G. P. Applications of Nonlinear Fiber Optics; Academic Press: Boston, 2007.

[2] Marshall, J.; Stewart, G.; Whitenett, G. Design of a tunable L-band multi-wavelength laser system for application to gas spectroscopy. Meas. Sci. Technol. 2006, 17, 1023-1031.

[3] Okhotnikov, O.; Grudinin, A.; Pessa, M. Ultra-fast fibre laser systems based on SESAM technology: New horizons and applications. New J. Phys. 2004, 6, 177.

[4] Nelson, L. E.; Jones, D. J.; Tamura, K.; Haus, H. A; Ippen, E. P. Ultrashort-pulse fiber ring lasers. Appl. Phys. B 1997, 65, 277-294.

[5] Fermann, M. E.; Galvanauskas, A.; Sucha, G.; Harter, D. Fiber-lasers for ultrafast optics. Appl. Phys. B 1997, 65, 259-275.

[6] Keller, U. Ultrafast solid-state lasers. In Progress in Optics. Wolf, E. Ed.; Elsevier: Amsterdam, 2004.

[7] Hasan, T.; Sun, Z. P.; Wang, F.; Bonaccorso, F.; Tan, P. H.; Rozhin, A. G.; Ferrari, A. C. Nanotube-polymer composites for ultrafast photonics. Adv. Mater. 2009, 21, 3874-3899.

[8] Sun, Z. P.; Hasan, T.; Torrisi, F.; Popa, D.; Privitera, G.; Wang, F. Q.; Bonaccorso, F.; Basko, D. M.; Ferrari, A. C. Graphene mode-locked ultrafast laser. ACS Nano 2010, 4, 803-810.

[9] Set, S. Y.; Yaguchi, H.; Tanaka, Y.; Jablonski, M. Ultrafast fiber pulsed lasers incorporating carbon nanotubes. IEEE $J$. Sel. Top. Quantum Electron. 2004, 10, 137-146.

[10] Nakazawa, M.; Nakahara, S.; Hirooka,T.; Yoshida, M.; Kaino, T.; Komatsu, K. Polymer saturable absorber materials in the $1.5 \mu \mathrm{m}$ band using poly-methyl-methacrylate and polystyrene 
with single-wall carbon nanotubes and their application to a femtosecond laser. Opt. Lett. 2006, 31, 915-917.

[11] Scardaci, V.; Rozhin, A. G.; Hennrich, F.; Milne, W. I.; Ferrari, A. C. Carbon nanotube-polymer composites for photonic devices. Physica E 2007, 37, 115-118.

[12] Song, Y. W.; Yamashita, S.; Maruyama, S. Single-walled carbon nanotubes for high-energy optical pulse formation. Appl. Phys. Lett. 2008, 92, 021115.

[13] Rozhin, A. G.; Scardaci, V.; Wang, F.; Hennrich, F.; White, I. H.; Milne, W. I.; Ferrari, A. C. Generation of ultra-fast laser pulses using nanotube mode-lockers. Phys. Status Solidi B 2006, 243, 3551-3555.

[14] Sun, Z.; Rozhin, A. G.; Wang, F.; Scardaci, V.; Milne, W. I.; White, I. H.; Hennrich, F.; Ferrari, A. C. L-band ultrafast fiber laser mode locked by carbon nanotubes. Appl. Phys. Lett. 2008, 93, 061114.

[15] Scardaci, V.; Sun, Z.; Wang, F.; Rozhin, A. G.; Hasan, T.; Hennrich, F.; White, I. H.; Milne, W. I.; Ferrari, A. C. Carbon nanotube polycarbonate composites for ultrafast lasers. $A d v$. Mater. 2008, 20, 4040-4043.

[16] Wang, F.; Rozhin, A. G.; Scardaci, V.; Sun, Z.; Hennrich, F.; White, I. H.; Milne, W. I.; Ferrari, A. C. Widebandtuneable, nanotube mode-locked, fibre laser. Nat. Nanotechnol. 2008, 3, 738-742.

[17] Kelleher, E. J. R.; Travers, J. C.; Sun, Z.; Rozhin, A. G.; Ferrari, A. C.; Popov, S. V.; Taylor, J. R. Nanosecond-pulse fiber lasers mode-locked with nanotubes. Appl. Phys. Lett. 2009, 95, 111108.

[18] Sun, Z.; Rozhin, A. G.; Wang, F.; Hasan, T.; Popa, D.; O’Neill, W.; Ferrari, A. C. A compact, high power, ultrafast laser mode-locked by carbon nanotubes. Appl. Phys. Lett. 2009, 95, 253102.

[19] Solodyankin, M. A.; Obraztsova, E. D.; Lobach, A. S.; Chernov, A. I.; Tausenev, A. V.; Konov, V. I.; Dianov, E. M. Mode-locked $1.93 \mu \mathrm{m}$ thulium fiber laser with a carbon nanotube absorber. Opt. Lett. 2008, 33, 1336-1338.

[20] Nicholson, J. W.; Windeler, R. S.; DiGiovanni, D. J. Optically driven deposition of single-walled carbon-nanotube saturable absorbers on optical fiber end-faces. Opt. Express 2007, 15, 9176-9183.

[21] Kieu, K.; Wise, F. W. All-fiber normal-dispersion femtosecond laser. Opt. Express 2008, 16, 11453-11458.

[22] Shohda, F.; Shirato, T.; Nakazawa, M.; Komatsu, K.; Kaino, T. A passively mode-locked femtosecond soliton fiber laser at $1.5 \mu \mathrm{m}$ with a CNT-doped polycarbonate saturable absorber. Opt. Express 2008, 16, 21191-21198.

[23] Senoo, Y.; Nishizawa, N.; Sakakibara, Y.; Sumimura, K.; Itoga, E.; Kataura, H.; Itoh, K. Polarization-maintaining, high-energy, wavelength-tunable, Er-doped ultrashort pulse fiber laser using carbon-nanotube polyimide film. Opt. Express 2009, 17, 20233-20241.

[24] Della Valle, G.; Osellame, R.; Galzerano, G.; Chiodo, N.; Cerullo, G.; Laporta, P.; Svelto, O.; Morgner, U.; Rozhin, A. G.; Scardaci, V.; Ferrari, A. C. Passive mode locking by carbon nanotubes in a femtosecond laser written waveguide laser. Appl. Phys. Lett. 2006, 89, 231115.

[25] Fong, K. H.; Kikuchi, K.; Goh, C. S.; Set, S. Y.; Grange, R.; Haiml, M.; Schlatter, A.; Keller, U. Solid-state Er:Yb:glass laser mode-locked by using single-wall carbon nanotube thin film. Opt. Lett. 2007, 32, 38-40.

[26] Schibli, T. R.; Minoshima, K.; Kataura, H.; Itoga, E.; Minami, N.; Kazaoui, S.; Miyashita, K.; Tokumoto, M.; Sakakibara, Y. Ultrashort pulse-generation by saturable absorber mirrors based on polymer-embedded carbon nanotubes. Opt. Express 2005, 13, 8025-8031.

[27] Schmidt, A.; Rivier, S.; Steinmeyer, G.; Yim, J. H.; Cho, W. B.; Lee, S.; Rotermund, F.; Pujol, M. C.; Mateos, X.; Aguilo, M.; Diaz, F.; Petrov, V.; Griebner, U. Passive mode locking of $\mathrm{Yb}: \mathrm{KLuW}$ using a single-walled carbon nanotube saturable absorber. Opt. Lett. 2008, 33, 729-731.

[28] Song, Y. W.; Yamashita, S.; Goh, C. S.; Set, S. Y. Passively mode-locked lasers with 17.2-GHz fundamental-mode repetition rate pulsed by carbon nanotubes. Opt. Lett. 2007, 32, 430-432.

[29] Tamura, K.; Ippen, E. P.; Haus, H. A.; Nelson, L. E. 77-fs pulse generation from a stretched-pulse mode-locked all-fiber ring laser. Opt. Lett. 1993, 18, 1080-1082.

[30] Haus, H. A.; Tamura, K.; Nelson, L. E.; Ippen, E. P. Stretched-pulse additive-pulse mode-locking in fiber ring lasers-Theory and experiment. IEEE J. Quantum Electron. 1995, 31, 591-598.

[31] Tamura, K.; Nelson, L. E.; Haus, H. A.; Ippen, E. P. Soliton versus nonsoliton operation of fiber ring lasers. Appl. Phys. Lett. 1994, 64, 149-151.

[32] Ilday, F. O.; Wise, F. W.; Sosnowski, T. High-energy femtosecond stretched-pulse fiber laser with a nonlinear optical loop mirror. Opt. Lett. 2002, 27, 1531-1533.

[33] Lefort, L.; Price, J. H. V.; Richardson, D. J.; Spuhler, G. J.; Paschotta, R.; Keller, U.; Fry, A. R.; Weston, J. Practical low-noise stretched-pulse $\mathrm{Yb}^{3+}$-doped fiber laser. Opt. Lett. 2002, 27, 291-293.

[34] O’Connell, M. J.; Bachilo, S. M.; Huffman, C. B.; Moore, V. C.; Strano, M. S.; Haroz, E. H.; Rialon, K. L.; Boul, P. J.; Noon, W. H.; Kittrell, C.; Ma, J. P.; Hauge, R. H.; Weisman, R. B.; Smalley, R. E. Band gap fluorescence from individual single-walled carbon nanotubes. Science 2002, 297, 593-596.

[35] Moore, V. C.; Strano, M. S.; Haroz, E. H.; Hauge, R. H.; Smalley, R. E. Individually suspended single-walled carbon 
nanotubes in various surfactants. Nano Lett. 2003, 3, 1379-1382.

[36] Haggenmueller, R.; Rahatekar, S. S.; Fagan, J. A.; Chun, J. H.; Becker, M. L.; Naik, R. R.; Krauss, T.; Carlson, L.; Kadla, J. F.; Trulove, P. C., et al. Comparison of the quality of aqueous dispersions of single wall carbon nanotubes using surfactants and biomolecules. Langmuir 2008, 24, 5070-5078.

[37] Hasan, T; Tan, P. H.; Bonaccorso, F; Rozhin, A. G.; Scardaci, V.; Milne, W. I.; Ferrari, A. C. Polymer-assisted isolation of single wall carbon nanotubes in organic solvents for optical-quality nanotube polymer composites. J. Phys. Chem. C 2008, 112, 20227-20232.

[38] Bandyopadhyaya, R.; Nativ-Roth, E.; Regev, O.; YerushalmiRozen, R. Stabilization of individual carbon nanotubes in aqueous solutions. Nano Lett. 2002, 2, 25-28.

[39] Minami, N.; Kim, Y. J.; Miyashita, K.; Kazaoui, S.; Nalini, B. Cellulose derivatives as excellent dispersants for singlewall carbon nanotubes as demonstrated by absorption and photoluminescence spectroscopy. Appl. Phys. Lett. 2006, 88, 093123.

[40] Birkin, P. R.; Offin, D. G.; Joseph, P. F.; Leighton, T. G. Cavitation, shock waves and the invasive nature of sonoelectrochemistry. J. Phys. Chem. B 2005, 109, 16997-17005.

[41] Lebedkin, S.; Schweiss, P.; Renker, B.; Malik, S.; Hennrich, F.; Neumaier, M.; Stoermer, C.; Kappes, M. M. Single-wall carbon nanotubes with diameters approaching $6 \mathrm{~nm}$ obtained by laser vaporization. Carbon 2002, 40, 417-423.

[42] Hennrich, F.; Wellmann, R.; Malik, S.; Lebedkin, S.; Kappes, M. M. Reversible modification of the absorption properties of single-walled carbon nanotube thin films via nitric acid exposure. Phys. Chem. Chem. Phys. 2003, 5, 178-183.

[43] Piscanec, S.; Lazzeri, M.; Robertson, J.; Ferrari, A. C.; Mauri,
F. Optical phonons in carbon nanotubes: Kohn anomalies, Peierls distortions, and dynamic effects. Phys. Rev. B 2007, 75, 035427.

[44] Piscanec, S.; Lazzeri, M.; Mauri, F.; Ferrari, A. C.; Robertson, J. Kohn anomalies and electron-phonon interactions in graphite. Phys. Rev. Lett. 2004, 93, 185503.

[45] Telg, H.; Maultzsch, J.; Reich, S.; Hennrich, F.; Thomsen, C. Chirality distribution and transition energies of carbon nanotubes. Phys. Rev. Lett. 2004, 93, 177401.

[46] Telg, H.; Maultzsch, J.; Reich, S.; Thomsen, C. ResonantRaman intensities and transition energies of the $E_{11}$ transition in carbon nanotubes. Phys. Rev. B 2006, 74, 115415.

[47] Fantini, C.; Jorio, A.; Souza, M.; Strano, M. S.; Dresselhaus, M. S.; Pimenta, M. A. Optical transition energies for carbon nanotubes from resonant Raman spectroscopy: Environment and temperature effects. Phys. Rev. Lett. 2004, 93, 147406.

[48] Kataura, H.; Kumazawa, Y.; Maniwa, Y.; Umezu, I.; Suzuki, S.; Ohtsuka, Y.; Achiba, Y. Optical properties of single-wall carbon nanotubes. Synth. Met. 1999, 103, 2555-2558.

[49] Dennis, M. L.; Duling, I. N. Experimental study of sideband generation in femtosecond fiber lasers. IEEE J. Quantum Electron. 1994, 30, 1469-1477.

[50] Koechner, W. Solid-State Laser Engineering; Springer: Berlin,1999.

[51] Spielmann, C.; Curley, P. F.; Brabec, T.; Krausz, F. Ultrabroadband femtosecond lasers. IEEE J. Quantum Electron. 1994, 30, 1100-1114.

[52] Agrawal, G. P. Nonlinear Fiber Optics; Academic Press: San Diego, 2001.

[53] von der Linde, D. Characterization of the noise in continuously operating mode-locked lasers. Appl. Phys. B 1986, 39, 201-217. 